\title{
Tracheal tube stylets as a habit
}

\author{
Marco Gemma $^{1}$
}

Received: 29 November 2016 / Accepted: 12 January 2017 / Published online: 25 January 2017

(c) Springer Science+Business Media Dordrecht 2017

\section{Dear Sir,}

I read with interest the recent review by Grape and Schoettke about "The role of tracheal tube introducers and stylets in current airway management" [1] and I agree with the Authors' analysis and conclusions.

In addressing the issue of stylet use, the Authors state that "As no studies have proven a benefit on success rate or diminished tracheal intubation attempts, these types of stylets have not gained widespread use." Unfortunately this statement is not universally true. In fact, with respect to this topic, I wish to draw attention to a frequent malpractice I often witnessed in routine anesthesia care. In my experience, besides any published guideline and written statement, the routine use of stylets for elective tracheal intubation in the first place (at the first attempt and without having experienced any actual difficulty) is common practice in several hospitals. I would strongly oppose this practice anytime I come across it.

It should be clearly stated that stylets are useful and often life-saving devices, but that they should be devoted to the difficult intubation scenario and are not intended for routine use.

Best regards.

Compliance with Ethical Standards

I hereby state that

- the present Letter does not involve any research on humans or animals.

- no informed consent is needed.

- I have no conflict of interest with respect to the content of this Letter.

\section{References}

1. Grape S, Schoettke P. The role of tracheal tube introducers and stylets in current airway management. J Clin Monit Comput. 2016. doi:10.1007/s10877-016-9879-8.
Marco Gemma

gemma.marco@hsr.it

1 S. Raffaele Hospital, Milano, Italy 